\title{
Preon Prophecies by the Standard Model
}

\author{
Sverker Fredriksson \\ Department of Physics, Luleå University of Technology, SE-97187 Luleå, Sweden
}

\begin{abstract}
The Standard Model of quarks and leptons is, at first sight, nothing but a set of ad hoc rules, with no connections, and no clues to their true background. At a closer look, however, there are many inherent prophecies that point in the same direction: Compositeness in terms of three stable preons.
\end{abstract}

\section{Introduction}

The Standard Model (SM) of quarks and leptons serves as a bible of high-energy physics. It even resembles the real Bible, in the sense that it is mainly a set of "stories", rules and wisdoms, which reflect both real life and human thinking.

The SM is also flexible, since it can easily be complemented with new findings or ideas, such as neutrino oscillations, supersymmetry, or new fundamental quarks and leptons, just like the first Bible was once complemented with the New Testament by Christians. Unlike the Bible, the SM does not give a clue to its deeper meaning, nor does it reveal any connections between its many bits and pieces. The SM is hence a quantitative success, but a qualitative mess.

What I will argue in this talk, however, is that the situation is not that unclear or hopeless. Rather, the SM is full of prophecies and hints to its deeper background, many of which can be understood after a deeper look into those SM features that have been put in entirely by hand. I will make frequent references to the "History Book", in order to investigate if such ideas or situations have appeared before. As you will see, there have been numerous situations in the past when paradoxes and problems like the ones in the SM turned out to come from compositeness of the "fundamental" particles of those days.

To be more precise, I will discuss the following aspects of the SM:

- there are six quarks and six leptons in three families

- most quarks and leptons are unstable

- there are several ad hoc quantum numbers

- some quarks, leptons and gauge bosons mix/oscillate

- the heavy vector bosons are massive and unstable

- the $Z^{0}$ mixes with the photon

The discussion will converge toward more precise features of a preon model, and I will conclude by briefly describing a recent one, presented by Jean-Jacques Dugne, Johan Hansson and myself [1]. The discussion presented here is, in fact, an offspring of our work with that particular model. 


\section{Some General Observations}

Before going into some detail with preons and the SM, a few more general observations can be made. First of all, the History Book tells us that "there is always a deeper layer of compositeness". Earlier layers have normally been suggested and/or discovered when the model in fashion became too complex, or when there were too many different models for the "fundamental" particles. Such leaps to new levels of compositeness come every $30-40$ years, which means that time is ripe for preons!

Nevertheless, compositeness has never been a main trend of high-energy research. Rather, an overwhelming majority of theorists has traditionally regarded the elementary particles of the day as the fundamental ones.

Compositeness usually comes into fashion as a viable theory only if strongly supported by experiment, or if suggested by some well-known theorist. In the latter case, the interest fades away after some time, if not supported by observations. Examples are the quark model by Gell-Mann and Zweig [23] and the early preon models by Harari 4] and Shupe [5] ("rishons") and by Fritzsch and Mandelbaum [6] ("haplons").

The quark model gained a wider acceptance only after the pioneering deepinelastic scattering data of the late 1960s and the early 1970s, and their interpretation in terms of partons. There are no such supporting data for preons, and the interest in the first preon models was therefore gone a long time ago. The concept of compositeness still appears now and then in experimental work, but mostly in routine searches for deviations from the SM predictions in events with large transferred momenta. The lack of signals are normally quoted as a minimal preon energy scale of a few $\mathrm{TeV}$ [7. It is to be understood as an inverse length-scale in a hypothetical form factor of internal preon wave functions (and not as a preon mass scale). In order to restrict this length-scale further, one would seemingly need the high energies of the upcoming CERN LHC facility, or at least high-statistics data from RUN II of the Fermilab Tevatron. However, this does not exclude that preons could reveal themselves in other ways, e.g., at the Tevatron, such as through the discovery of new, exotic quarks or leptons. An earlier example was the discovery of the exotic $\Omega^{-}$hyperon and its importance for the subsequent quark model.

\section{Too Many Quarks and Leptons}

The most common argument in favour of preons $[\underline{8}$ is that there are too many quarks and leptons to let any SM enthusiast feel comfortable. There is no obvious logical reason why there would be (at least) twelve fundamental particles. The case for preons is strengthened by the fact that these particles fall into a nice pattern of three "families".

The History Book tells us that the existence of too many fundamental particles in the past always preceded the discovery of yet smaller and fewer building blocks (not counting the era of "earth, fire, air and water"). 


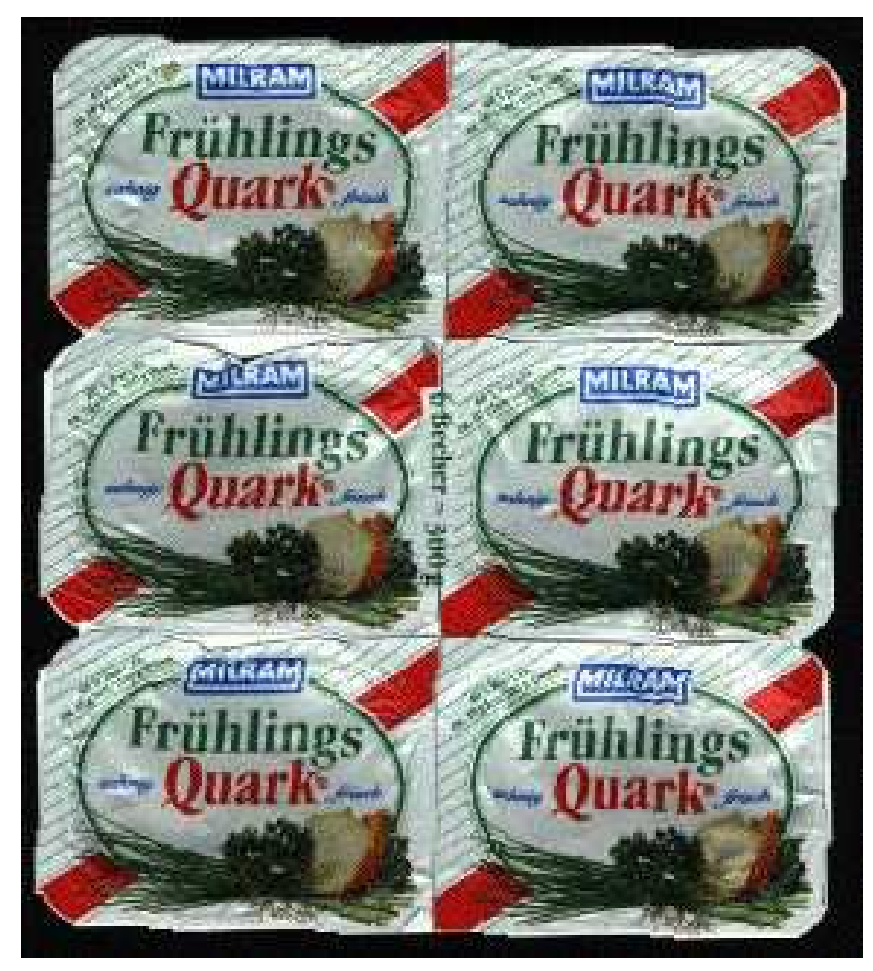

Fig. 1. Why do quarks come in six-pack, like in German supermarkets?

\section{Examples are:}

- the existence of too many elements was explained by the atoms consisting of electrons and nuclei;

- the existence of many isotopes of one and the same element is a by-product of the compositeness of nuclei, containing protons and neutrons;

- the hundreds of hadrons that puzzled high-energy physicists a half-century ago reflect their quark structure. The patterns among the early hadrons led the mind to the number three, and resulted in a model with three different quarks, with a quark-flavour $S U(3)$ symmetry.

A first conclusion is therefore that the many quarks and leptons reflect a preon substructure, and that the pattern of three families comes from the existence of three preons, with a preon-flavour $S U(3)$ symmetry. It is noteworthy that the early preon models did not, in general, provide a more rational explanation of the quarks and leptons of those days. Typically, four different preons were used to explain the existence of the four particles of the lightest quark/lepton family, while the heavier ones were not properly understood. A good preon model should hence explain all quarks and leptons on equal terms, at least in some sense. 


\section{$4 \quad$ Unstable Fundamental Particles?}

Most quarks and leptons are unstable, decaying into lighter ones, until only stable (?) electrons, protons and neutrinos remain. In my view there is a logical problem with unstable fundamental particles: How can Nature's most fundamental objects decay into equally fundamental objects? As to the best of my knowledge, this simple argument has not been debated earlier in the literature. When discussing this with colleagues, I am often met by the attitude that Nature is anyway so strange, so why bother?

Again the History Book gives some moral support to the preon solution, because all decays of previous "fundamental" particles have mirrored their compositeness. Examples are:

- the decays of atoms were found to come from the decays of their nuclei;

- which were later blamed on the decays of their nucleons;

- which were later blamed on the decays of their quarks.

So, where will this sequence of explanations end? In my opinion, not until we find a level with stable constituents. A preon model must therefore either have absolutely stable preons, or rely on yet unstable and composite preons ("prepreons"). If preons are stable, all quark and lepton decays are just regrouping of preons into systems with lighter quarks and leptons. This strongly limits the number of possible preon models. The pioneering preon models quoted above did not have this property. The heavier two families either remained unexplained, or were assumed to be internal excitations of the lightest one, decaying to their own ground states.

Summing up the prophecies so far, one can envisage the existence of three absolutely stable preons. This makes net preon flavour a completely conserved quantity in all particle reactions. A by-product is that in case two quarks or leptons would have identical net preon flavours, they will (or must) mix into new mass eigenstates. This would be equivalent to, e.g., $\rho / \omega$ - and $\eta / \eta^{\prime}$-mixing in the quark model. Hence such a preon model provides a possibility to understand the various mixings of quarks, neutrinos and weak isospin eigenstates in the SM. These aspects will be discussed later.

\section{$5 \quad$ Ad hoc Quantum Numbers}

The SM contains a few quantum numbers that have been put in by hand, without a deeper understanding, and just in order to describe that some quark and lepton quantities are conserved or partially conserved.

These are quantum numbers that either seem absolutely conserved, such as baryon number, or are known to be conserved only in some reactions, e.g., weak isospin. Lepton numbers form a grey-zone. They seemed to be absolutely conserved until quite recently, when neutrino oscillations were discovered.

The History Book again gives some clues. A long time ago, the "isotopic numbers" were understood only after the discovery of the neutron and the compositeness of atomic nuclei. In the 1950s hadronic isospin and strangeness/hypercharge 
were introduced to describe the observed approximate symmetries of hadronic decays and interactions. These three quantum numbers turned out to come from three quark flavours.

Since the number three pops up also in the SM, it is tempting to guess that lepton number conservation has to do with preon number conservation, i.e., preon-flavour $S U(3)$ symmetry. The disturbing neutrino oscillations/mixings can then be understood if two or more neutrinos have identical net preon flavour. This will be discussed in the next Section. The connection between weak isospin and the number of preons is not as obvious, but will also be discussed below.

\section{Oscillations and Mixings of Fundamental Particles?}

The SM prescribes that certain created quarks, leptons and gauge bosons mix into new eigenstates before being detected. The History Book tells about a few such situations in the past.

A classical example is that different isotopes, created in nuclear reactions, mix in Nature in certain proportions, and are inseparable in normal chemical reactions. Chemical isotope mixing hence has its root in the compositeness of atomic nuclei.

A more modern case is the mixing/oscillation of the two neutral kaons $K^{0}$ and $\bar{K}^{0}$ into the mass eigenstates $K_{L}$ and $K_{S}$. This comes about because of quark reactions inside the kaons, i.e., the compositeness of hadrons.

Both cases are examples of what happens when virtually different states have identical net quantum numbers relative the particular interaction used to "detect" them. I will now discuss three similar mixings of "fundamental" particles in the SM, and their interpretations in terms of compositeness.

\subsection{Example 1: The Cabibbo Mixing of $d$ and $s$}

Let us assume that the $d$ and $s$ quarks mix into the weak-interaction ("mass") eigenstates $d^{\prime}$ and $s^{\prime}$ because they have identical net preon contents. The question is then how this can be arranged in detail. There are in principle two different solutions. One is that the two quarks have identical preon contents, although with some internal differences between the detailed preon wave functions, e.g., with two different internal spin structures. The other one is to focus on the word "net", meaning that some preon flavours cancel inside either quark, e.g., because they contain preon-antipreon pairs.

It turns out that the first alternative fits the neutrino sector better (see below). I therefore suggest that a quark contains a preon-antipreon pair plus an additional preon. As an example, consider a situation where the two quarks have the following compositions: $d=\beta \bar{\beta} \bar{\delta}$ and $s=\alpha \bar{\alpha} \bar{\delta}$, both with the net flavour of the $\bar{\delta}$ preon. If we assume that quark production in strong (QCD) processes always starts with pure preon states, these two quarks will subsequently mix into mass eigenstates before they decay, or take part in a weak process. If looked upon as an oscillation, the mixing can be illustrated with the Feynman-like diagram of 
Fig. 2. A preon-antipreon pair inside a quark can annihilate and produce another pair, thereby forming another quark.

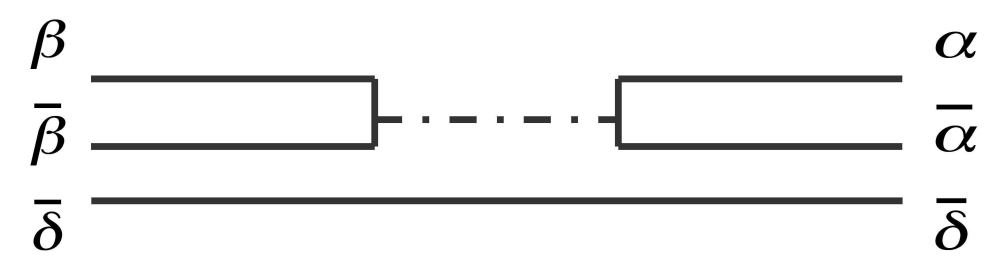

Fig. 2. One way for two composite quarks to mix quantum-mechanically. A preonantipreon pair annihilates and turns into another pair

For technical reasons I have used the convention of an antipreon $(\bar{\delta})$ for the common preon of $d$ and $s$. It is naturally not clear in detail how one preon pair converts to another. The intermediate system can be one or more photons, one or more gluons, or maybe some new gauge bosons ("hypergluons").

Neither does it seem realistic that one and the same phenomenon can explain all quark mixings of the so-called CKM matrix 910. There is simply no third state made up of these three preons that can oscillate in a similar way to a $d$ or an $s$. On the other hand, the smaller CKM matrix elements differ by an order of magnitude from the Cabibbo one, indicating that the detailed mechanism here is different from the one of Fig. 2 for $d$ and $s$. I refer to [1] for a preon-based discussion of other quark mixings than the $d / s$ one.

The preon charges can easily be chosen as to fit the charge of the two quarks. Obviously, $\delta$ must have charge $+e / 3$, while the other two can be "anything". It is wise to choose the charges $+e / 3$ also for $\alpha$ and $-2 e / 3$ for $\beta$. This will be obvious from the discussion in 6.2 below, although it already now seems attractive to mimic the three charges of the original flavour-SU(3) quark model.

\subsection{Example 2: Neutrino Mixing}

Once we choose preon charges as multiples of $e / 3$, and prescribe that a quark contains three entities, it becomes almost necessary to build the integer-charged leptons as three-preon states. With the charges defined above there are just three main ways to make neutrinos: $\alpha \beta \delta, \alpha \alpha \beta$ and $\delta \delta \beta$.

The three preons build up a total spin $1 / 2$, which means that there are several possible spin combinations. For simplicity, let us assume that two unequal preons prefer to form a total spin-0 "dipreon" pair, in the same fashion as quarks tend to pair up in diquarks in many situations [11. Such restrictions result in the following five neutrinos: $\nu_{1}=\alpha(\beta \delta)_{S=0}, \nu_{2}=\beta(\alpha \delta)_{S=0}, \nu_{3}=\delta(\beta \alpha)_{S=0}$. $\nu_{4}=\alpha(\beta \alpha)_{S=0}, \nu_{5}=\delta(\beta \delta)_{S=0}$. Note the similarity with the five neutral baryons in the spin- $1 / 2$ nonet (octet + singlet) of the original quark model.

It can be seen that the three neutrinos $\nu_{1}, \nu_{2}$ and $\nu_{3}$ indeed have identical net preon flavours, and hence can mix/oscillate into three new mass eigenstates. 
Such an oscillation can be seen as one of the three preons oscillating between the two spin-0 pairs that it can form with the other two preons. Figure 3 illustrates the situation for an oscillation between $\nu_{1}$ and $\nu_{2}$. At this primitive stage of the discussion it is not possible to pinpoint the actual neutrinos that correspond to the different preon states. Three of the five states must naturally be connected to the three known neutrinos, while the other two must have masses in excess of half the $Z^{0}$ mass.

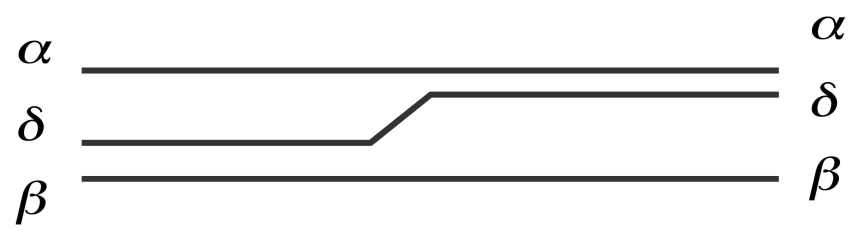

Fig. 3. One way for two composite neutrinos to mix/oscillate. A preon oscillates in and out of two different "dipreon" (spin-0) pairs

It can be added that with these particular preons it is impossible to construct charged leptons that would oscillate or mix in the same way. Neither can there be decays of the type $\mu \rightarrow e \gamma$.

\subsection{Example 3: Electroweak Mixing of $W^{0}$ and $B^{0}$}

In many preon models 8 the weak gauge bosons are supposed to be composite and built up by preon-antipreon pairs in total spin 1 . With the preons described earlier, it is tempting to define $W^{+}=(\alpha \bar{\beta})$ and $W^{-}=(\beta \bar{\alpha})$. The neutral sector is more complicated. There are five neutral combinations of the three preons, and three of these have identical net preon numbers: $\alpha \bar{\alpha}, \beta \bar{\beta}$ and $\delta \bar{\delta}$. They resemble the $\rho^{0}, \omega$ and $\phi$ of the vector meson nonet, and are expected to mix. Looking closer into the preon-flavour $S U(3)$ structure, it seems as if some (wavefunction) combinations correspond to the two neutral weak-isospin eigenstates, namely $W^{0}=(\alpha \bar{\alpha}-\beta \bar{\beta}) / \sqrt{2}$ and $B^{0}=(\alpha \bar{\alpha}+\beta \bar{\beta}) / \sqrt{2}$. These two then mix into two mass eigenstates, $Z^{0}$ and $Z^{\prime}$ (a new and even heavier boson). Note that the mixing partner of the normal $Z$ is not the photon, like in the SM. The reason is that the weak interaction is not fundamental in models with composite $Z \mathrm{~s}$ and $W \mathrm{~s}$, and there can hence not be an electroweak unification (and no Higgs!). This aspect will be further discussed later.

The mixing of $(\alpha \bar{\alpha})$ with $(\beta \bar{\beta})$ can again can be illustrated in the fashion of a Feynman diagram, as in Fig. 4.

The intermediate state must now be neutral in both charge and colour, as well as have spin 1. It could hence be one or more photons, two or three gluons, or a number of hypothetical "hypergluons". 


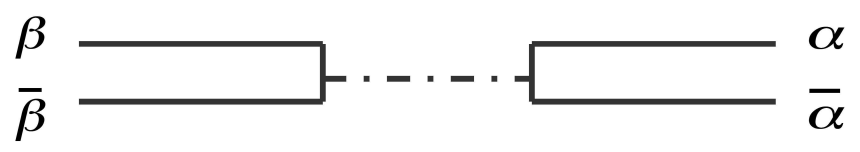

Fig. 4. One way for two composite neutral vector bosons to mix. A preon-antipreon pair annihilates and turns into another pair

\subsection{An Interesting Comparison}

A closer look shows that the action in Figs. $2 \& 4$ takes place through very similar preon subprocesses, as shown again in Fig. 5.

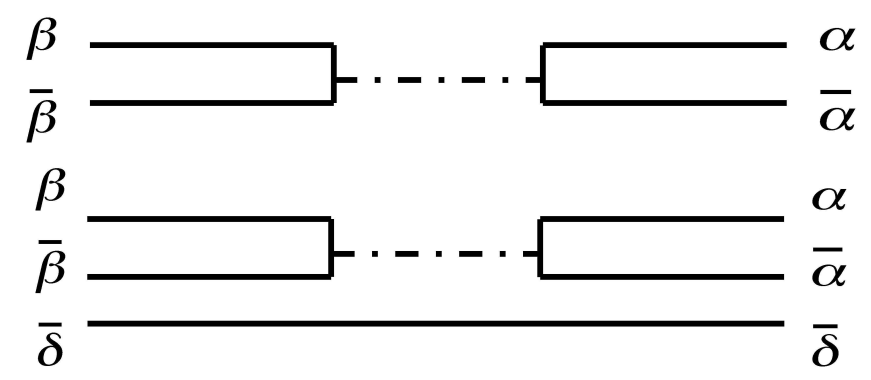

Fig. 5. The Weinberg mixing of $W^{0}$ and $B^{0}$, in the upper part of the figure, and the Cabibbo mixing of $d$ and $s$, in the lower part, come from the same basic preon processes, and are hence related

If the mixing fraction of the $(\alpha \bar{\alpha})$ and $(\beta \bar{\beta})$ pairs depends only on the masses and/or electric charges of $\alpha$ and $\beta$, and not on, e.g., the systems they sit in, or their total colour or spin, then one can show that there is a relation between the Cabibbo and Weinberg angles [1:

$$
\cos \theta_{W}-\sin \theta_{W}=\sqrt{2} \sin \theta_{C}
$$

With $\sin ^{2} \theta_{W}=0.23117 \pm 0.00016$ and $\sin \theta_{C}=0.2225 \pm 0.0035$ [7] the lhs $=$ $0.396 \pm 0.001$ and the $r h s=0.315 \pm 0.005$. This is a fair agreement considering the rough assumptions. In addition, if we simplify the situation further and assume that the mixings occur through one-photon intermediate states, then the preon-antipreon pairs occur in proportion to their squared charges, and we expect:

$$
\sin \theta_{C}=q_{\alpha}^{2} /\left(q_{\alpha}^{2}+q_{\beta}^{2}\right)=1 / 5,
$$

which again is not far from reality. 


\section{Massive, Charged and Unstable Weak Gauge Bosons}

Most ad hoc SM assumptions that lack a deeper background have to do with the ugly fact that the weak interaction has massive gauge bosons. That is the excuse for introducing the Higgs mechanism with all its inherent problems and unsatisfactory logic. This is typical for many of the odd features of the SM. They are introduced to cure and/or quantify earlier theoretical problems, rather than to explain the observationes from more basic principles. Ofter the cure causes side-effects, which need new cures, etc.

The situation with massive and unstable "gauge bosons" is not new in highenergy physics. In nuclear physics it is often productive to regard the vector mesons, $\rho, \omega, \phi$ etc, as gauge bosons of the nuclear forces that keep nucleons in place. They are good phenomenological couriers of QCD, by leaking colourneutral quark-antiquark pairs between nucleons. It seems as if spin-1 particles are better than scalar ones in faking true (massless) gauge bosons.

The History Book therefore warns us that massive gauge bosons are not fundamental, but some kind of "neutral" leakage of more basic forces. Hence $W$ and $Z$ might well be false gauge bosons that just leak the basic preon forces.

It would imply that there are nine different heavy vector bosons (if we believe in preon-flavour $S U(3))$. Hence six very heavy ones are yet to be discovered. Five of the bosons are neutral $(Z \mathrm{~s})$ and four charged $(W \mathrm{~s})$. Three of the $Z \mathrm{~s}$ have identical net preon flavour and would mix, since they are built up by $\alpha \bar{\alpha}, \beta \bar{\beta}$ and $\delta \bar{\delta}$. The remaining two $Z$ s are mutual antiparticles, being $\alpha \bar{\delta}$ and $\delta \bar{\alpha}$.

\section{Electroweak Unification and $\gamma / Z$ Mixing}

It remains to discuss why $Z^{0}$ seems to mix with the photon, in a way that is well described by the electroweak sector of the SM and parametrized by the Weinberg angle.

The History Book again tells why, because the situation is not new. In the 1960s it was discovered that the photon sometimes behaves like a hadron in interactions with nucleons. It was suggested that its wave function has a hadronic component, consisting mainly of the $\rho$ meson, but with some fraction also of more massive spin- 1 mesons at higher $Q^{2}$ values. This idea was dubbed Vector Meson Dominance (VMD) 12. It was developed in great detail and used to understand, or at least describe, a wealth of data. It is still a viable model for the behaviour of virtual photons in medium- $Q^{2}$ reactions with hadrons. Hence a photon is believed to couple directly to a $\rho$, like in Fig. 6 , when interacting with a hadron.

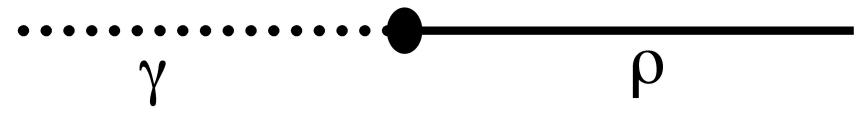

Fig. 6. The photon mixes with $\rho$ according to the Vector Meson Dominance model 
In the early days this was taken as a fact, and the origin of the coupling remained unknown for a while. A physicist with a gift to look into the future could then have come up with a radical idea: The photon and the $\rho$ are one and the same particle! This would lay the ground for a new theory of electrostrong unification, where the $\gamma / \rho$ mixing is parametrized by an electrostrong mixing angle. After a while the substantial difference in mass between the two particles would have inspired someone to invent an electrostrong Higgs boson. Or rather, a whole set of mixing angles and Higgs bosons, because the photon couples also to other vector mesons.

Why was this model never invented (except here)? Because soon after the birth of VMD, the quark model gained ground. Then it became evident that any spin-1 neutral meson couples to ("mixes" with) the photon just because its quarks are electrically charged. At high-enough $Q^{2}$ the photon sees the constituents, instead of the (invisible) neutral hadron. The true explanation of the $\gamma / \rho$ mixing is hence compositeness, as illustrated by Fig. 7 .

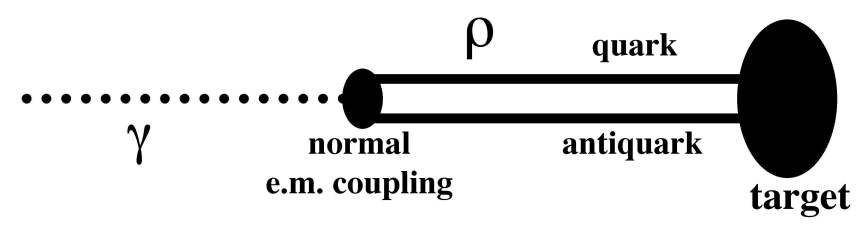

Fig. 7. The $\gamma / \rho$ mixing comes about because $\rho$ is composite

The conclusion for the electroweak sector and preons is then obvious. The $Z$ contains charged preons, and is hence destined to couple to a high- $Q^{2}$ photon, as in Fig. 8.

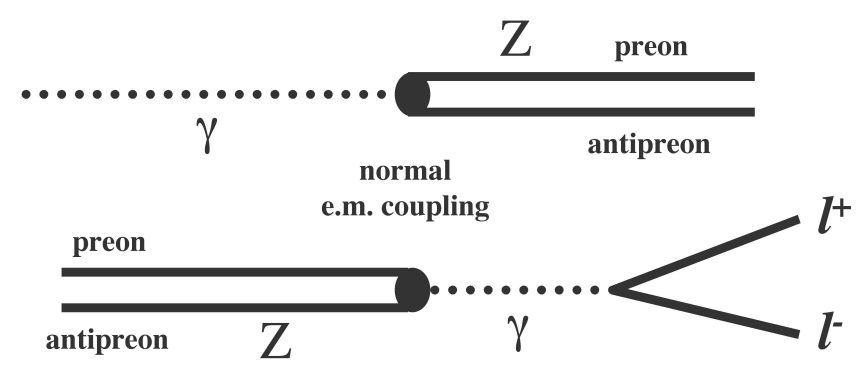

Fig. 8. The $\gamma / Z$ mixing comes about because $Z$ is composite

Qualitatively, the electroweak formalism is very similar to the old parametrization of the VDM model, i.e., the propagators in a Feynman-graph formalism look 
the same. The $Z$ and $W$ s are just very good at faking true gauge bosons. One can even understand why one and the same Weinberg angle would reasonably well describe not only the $\gamma / Z$ mixing in the SM but also the $Z / Z^{\prime}$ mixing in a preon model. This would come about if the conjectures leading to (1) and (2) were true, i.e., that the preon-antipreon states inside the various $Z$ s mix via virtual photons, rather than gluons or hypergluons.

However, the electroweak and electrostrong situation differ quantitatively, since no heavier $\left(Z^{\prime}\right)$ boson has revealed itself experimentally as deviations from the SM predictions, e.g., at the CERN LEP machine. Neither are there any traces of direct $Z^{\prime}$ production at the Fermilab Tevatron. The hypothetical $Z^{\prime}$ should therefore be much heavier than the $Z^{0}$ [7, unlike the vector-meson situation, where the $\omega$ is very close to $\rho$ in mass. This need not be troublesome for preons, because the preon- $S U(3)$ wave functions inside $Z \mathrm{~s}$ might differ substantially from the quark-SU(3) wave functions inside mesons.

It could be fruitful, however, to analyse the unconventional alternative that the $Z^{0}$ and the next $Z^{\prime}$ have almost equal masses. Maybe the $Z^{0}$ wave function is dominated by the $(\beta \bar{\beta})$ combination and the first $Z^{\prime}$ by $(\alpha \bar{\alpha})$. Then $e^{+} e^{-}$ annihilation would produce mainly the $Z^{0}$, for two reasons: (i) an annihilation to a photon would couple the photon four times as strongly to a $\beta \bar{\beta}$ pair than to an $\alpha \bar{\alpha}$ pair; (ii) an annihilation of only the dipreons in the $e^{+} e^{-}$pair would result in a $\beta \bar{\beta}$ system (see Table 2 below), which in turn would prefer to make a $Z^{0}$. At present it is unclear to me if a "hidden" $Z^{\prime}$, near the $Z^{0}$ in mass, would be in conflict with the CERN LEP precision tests of the SM.

\section{Conclusions: What next?}

This discussion of crucial ad hoc features of the standard model has, step-by-step, led to strict and rather detailed requirements of a hypothetical preon model for quarks, leptons and heavy vector bosons. As you might have guessed, they are all in line with the "preon-trinity" model presented earlier by Dugne, Hansson and myself [1].

The three preons have the charges given in Table 1. Also listed are the dipreons, necessary for understanding, e.g., neutrino oscillations. When entered as anti-dipreons, the scheme reveals a nice supersymmetric structure.

Table 1. A "supersymmetric" scheme of spin- $1 / 2$ preons and spin- 0 anti-dipreons.

\begin{tabular}{|c|c|}
\hline charge & $+e / 3-2 e / 3+e / 3$ \\
\hline $\begin{array}{r}\text { spin-1/2 } \\
\text { spin-0 (anti- }\end{array}$ & $\begin{array}{ccc}\alpha & \beta & \delta \\
(\bar{\beta} \bar{\delta}) & (\bar{\alpha} \bar{\delta}) & (\bar{\alpha} \bar{\beta})\end{array}$ \\
\hline
\end{tabular}

Following the SM prophecies, the leptons are now constructed as combinations of one preon and one dipreon, the quarks of one preon and one anti-dipreon, and the heavy vector bosons of one preon and one antipreon, as in Table 2. 
Table 2. The composite states in the preon-trinity model; leptons with one preon and one dipreon, quarks with one preon and one anti-dipreon, and heavy vector bosons with one preon and one antipreon.

\begin{tabular}{c|ccc|ccc|ccc|c} 
& $(\beta \delta)$ & $(\alpha \delta)$ & $(\alpha \beta)$ & $(\bar{\beta} \bar{\delta})$ & $(\bar{\alpha} \bar{\delta})$ & $(\bar{\alpha} \bar{\beta})$ & $\bar{\alpha}$ & $\bar{\beta}$ & $\bar{\delta}$ & \\
\hline$\alpha$ & $\nu_{e}$ & $\mu^{+}$ & $\nu_{\tau}$ & $u$ & $s$ & $c$ & $Z^{0}, Z^{\prime}$ & $W^{+}$ & $Z^{*}$ & $\alpha$ \\
$\beta$ & $e^{-}$ & $\bar{\nu}_{\mu}$ & $\tau^{-}$ & $d$ & $X$ & $b$ & $W^{-}$ & $Z^{\prime}, Z^{0}$ & $W^{\prime-}$ & $\beta$ \\
$\delta$ & $\nu_{\kappa 1}$ & $\kappa^{+}$ & $\nu_{\kappa 2}$ & $h$ & $g$ & $t$ & $\bar{Z}^{*}$ & $W^{\prime+}$ & $Z^{\prime \prime}, Z^{\prime}$ & $\delta$
\end{tabular}

The best way to test the model would be to search for the new (heavy) particles in Table 2. Since the top quark is most probably the one marked $t$, there is some hope that the missing quarks and leptons can be produced at least at the Fermilab Tevatron. I am now analysing the conjecture that all three quarks, $h, g$ and $t$, in Table 2 are hidden in the Tevatron data. The analyses of their decay modes are rather complex, especially in the light of the trigger and tagging conditions of the Tevatron experiments.

The $X$ quark is a mystery. It appears near the $d$ and $b$ in Table 2 , but it has charge $-4 e / 3$, since both its preon and anti-dipreon have charge $-2 e / 3$. This made us suspect 1 that the system is not bound, and hence not a real quark.

A final observation is that the heavy neutrino $\nu_{\kappa 2}$ in Table 2 can be produced in $e^{+} e^{-}$annihilation together with a normal antineutrino. We expect the $\nu_{\kappa 2}$ to be lighter than its related quark $(t)$, since quarks are in general heavier than the corresponding leptons. Then the $\nu_{\kappa 2}$ can, in principle, be produced at energies lower than around $175 \mathrm{GeV}$. Hence it might be visible in existing CERN LEP data. I am analysing the situation in some detail. All LEP groups have reported that there are no statistically significant signatures of decay products from a heavy neutrino ("neutral excited lepton"). So maybe a careful event-by-event check is the only hope of finding a hint of a heavy neutrino at LEP (or HERA).

\section{Acknowledgements}

First of all I would like to thank the Organisers for providing a wonderful atmosphere at Schlo $\beta$ Ringberg, and a most fantastic weather all over Bavaria. Remember that this praise comes right from the heart of a resident of the Swedish Arctic, where the ice on the Baltic broke up in early May, six weeks ago! I am also grateful for much advice from my preon partners Jean-Jacques Dugne and Johan Hansson. At an early stage this preon research was supported in part by the European Commission under contract CHRX-CT94-0450, within the network "The Fundamental Structure of Matter".

\section{References}

1. J.-J. Dugne, S. Fredriksson, J. Hansson: Europhys. Lett. 57, 188 (2002) 
2. M. Gell-Mann: Phys. Lett. 8, 214 (1964)

3. G. Zweig: report CERN-TH-412 (1964), unpublished

4. H. Harari: Phys. Lett. 86B, 83 (1979)

5. M.A. Shupe: Phys. Lett. 86B, 87 (1979)

6. H. Fritzsch, G. Mandelbaum: Phys. Lett. 102B, 319 (1981)

7. K. Hagiwara et al.: Review of Particle Physics, Phys. Rev. 57, 188 (2002)

8. I.A. D'Souza, C.S. Kalman: Preons (World Scientific, Singapore 1992)

9. N. Cabibbo: Phys. Rev. Lett. 10, 531 (1963)

10. M. Kobayashi, T. Maskawa: Prog. Theor. Phys. 49, 652 (1973)

11. For a review of diquarks, see M. Anselmino, E. Predazzi, S. Ekelin, S. Fredriksson, D.B. Lichtenberg: Rev. Mod. Phys. 65, 1199 (1993)

12. For a review of the early VMD model, see D. Schildknecht: Springer Tracts in Modern Physics 63, 57 (1972) 